Published in final edited form as:

J Assoc Inf Sci Technol. 2017 September ; 68(9): 2088-2100. doi:10.1002/asi.23855.

\title{
Measuring Text Difficulty Using Parse-Tree Frequency
}

\author{
David Kauchak ${ }^{1}$, Gondy Leroy ${ }^{2}$, and Alan Hogue ${ }^{3,4}$ \\ ${ }^{1}$ Computer Science Department, Pomona College, Claremont, CA \\ ${ }^{2}$ Department of Management Information Systems, Eller College of Management, University of \\ Arizona, Tucson, AZ \\ ${ }^{3}$ Department of Linguistics, University of Arizona, Tucson, AZ \\ ${ }^{4}$ Google Inc., Mountain View, CA
}

\begin{abstract}
Text simplification often relies on dated, unproven readability formulas. As an alternative and motivated by the success of term familiarity, we test a complementary measure: grammar familiarity. Grammar familiarity is measured as the frequency of the $3^{\text {rd }}$ level sentence parse tree and is useful for evaluating individual sentences. We created a database of $140 \mathrm{~K}$ unique $3^{\text {rd }}$ level parse structures by parsing and binning all 5.4M sentences in English Wikipedia. We then calculated the grammar frequencies across the corpus and created 11 frequency bins. We evaluate the measure with a user study and corpus analysis.

For the user study, we selected 20 sentences randomly from each bin, controlling for sentence length and term frequency, and recruited 30 readers per sentence $(\mathrm{N}=6,600)$ on Amazon Mechanical Turk. We measured actual difficulty (comprehension) using a Cloze test, perceived difficulty using a 5-point Likert scale, and time taken. Sentences with more frequent grammatical structures, even with very different surface presentations, were easier to understand, perceived as easier and took less time to read. Outcomes from readability formulas correlated with perceived but not with actual difficulty. Our corpus analysis shows how the metric can be used to understand grammar regularity in a broad range of corpora.
\end{abstract}

\section{Keywords}

Comprehension; Health Literacy; Patient Education; Text Simplification; Readability; Text Difficulty

\section{INTRODUCTION}

Creating readable and understandable text is critical in many domains, such as education, healthcare and legal materials, since text is one of the most common and cost-effect ways of disseminating information (Farmer et al., 2008). Readability formulas, such as the FleschKincaid grade level formula (Kincaid, Fishburne Jr, Rogers, \& Chissom, 1975), Simple

Corresponding Author: David Kauchak, Computer Science Department, 185 E 6th St, Pomona College, Claremont, CA 91711, Phone: 909-607-0473, david.kauchak@pomona.edu. 
Measure of Gobbledygook (SMOG) (McLaughlin, 1969) and Gunning-Fox index (Kim et al., 2007), are frequently used in many domains to create more understandable text. Unfortunately, there is little evidence that they are effective at increasing comprehension through their use. They use overly simple text statistics such as sentence length and the number of syllables to measure readability, which may correlate with readability level as assigned by experts, but do not translate into concrete methods for simplifying text and have not been provably shown to reliably produce simpler text (Bruce, Rubin, \& Starr, 1981; Connatser, 1999). Particularly in specialized domains, the underlying principles do not hold, e.g., in medicine shorter words are not always easier to understand: 'apnea' versus 'diabetes'. Simply using shorter sentences and words with fewer syllables does not make text more understandable and informative.

In previous work, we developed 'term familiarity', a new measure of word difficulty that leverages the Google Web Corpus to measure word difficulty. Based on this measure, we developed a semi-automated tool for lexical simplification. The tool identifies difficult terms and then suggests simpler variants based on ontologies and thesauri. The writer selects from these suggestions to make the simplification. Our approach was validated in user studies and shown to significantly decrease the difficulty of the text and improve user understanding (Leroy, Endicott, Kauchak, Mouradi, \& Just, 2013).

Motivated by these findings, we hypothesize that, not just at the word level, but more generally, phenomena that readers encounter more frequently are easier to understand. The foundation for this may lay in priming effects. In this paper, we examine how grammatical frequency impacts the difficulty of a sentence and introduce a new measure of sentence-level text difficulty based on the grammatical structure of the sentence. Specifically, we posit that the frequency of the parse tree structure of a sentence will impact the readability and the understandability of text; sentences that have grammatical structures that are more frequent (and therefore are more familiar) will be viewed as simpler and will be easier to understand. Unlike traditional readability measures, our approach can be applied naturally to single sentences as well as long texts and can provide concrete advice for simplification, i.e., changes to the grammatical structure. Additionally, grammar frequency is complementary to term familiarity; both the words that are used in a sentence as well as grammatical structure of the sentence impact comprehension.

Our approach is domain independent and only relies on a corpus of sentences, however, our long-term project application domain and the domain of our corpus study is the health and medical domain, so we briefly review the use of existing readability measures in that domain and compare our results with these. The medical domain is a particularly important application domain for readability measures and text simplification since it is estimated that around 90 million Americans do not have sufficient health literacy (Committee on Health Literacy - Institute of Medicine of the National Academies, 2004) and this deficiency costs the U.S. economy between $\$ 106$ billion and $\$ 238$ billion a year (Vernon, Trujillo, Rosenbaum, \& DeBuono, 2007). One of the key methods for remedying this disparity is identifying cost-effective and efficient methods for disseminating information. Currently, most methods focus on providing text that is suitable for reading by patients and health information consumers. The tools promoted and used for optimizing text difficulty are 
almost universally readability formulas. Their use is often encouraged in the medical profession by requirements to write and communicate at a $5^{\text {th }}$ or $6^{\text {th }}$ grade level (e.g., for informed consent with clinical trials) (Weiss, 2007).

The readability formulas are applied to patient education materials (Adkins \& Singh, 2001; Brandt, McCree, Lindley, Sharpe, \& Hutto, 2005), bereavement materials (Rathbun, Thornton, \& Fox, 2008), informed consent forms (Brainard, 2003) and even surveys (Maples, Franks, Stevens, \& Wallace, 2010). Unfortunately, while these readability formulas are commonly used, they have not been shown to positively impact teaching of the necessary information. Our work here makes a step towards better simplification tools by 1) introducing a sentence-level, data-driven approach for measuring the grammatical difficulty of a sentence and 2) specifically measuring the impact of this measure using both how difficult a sentence looks (perceived difficulty) as well as how difficult a sentence is to understand (actual difficulty). Few, if any, make this distinction.

\section{BACKGROUND}

To ensure a comprehensive and systematic approach to measuring text difficulty, many different types of features should be examined, e.g. lexical, syntactic and discourse. Each of these categories of text characteristics influences text difficulty in different ways and provide opportunities for simplification. In this work we focus on syntactic difficulty, though we briefly review here the role of words since they play a critical role in most simplification systems.

\section{The Role of Words in Simplification}

Words play a critical role in understanding. Texts that include words that a reader does not know will be more difficult to understand. Many early readability formulas tried to capture this and rely heavily on word characteristics to measure text difficulty (Kim et al., 2007; Kincaid et al., 1975; McLaughlin, 1969), e.g. the number of syllables (with the assumption that longer words are more difficult) or the presence of a word in a predefined word list (Bailin \& Grafstein, 2001). Recent approaches to predicting text difficulty that rely on trained models using labeled data find word-level features to be highly predictive of text difficulty (Collins-Thompson \& Callan, 2005; Leroy, Miller, Rosemblat, \& Browne, 2008; Pitler \& Nenkova, 2008).

The importance of individual words has also been shown in corpus studies that systematically compare vocabulary usage between texts of different difficulty levels. Comparing articles in Simple English Wikipedia—one of the largest corpora publicly available of simplified text-with those in English Wikipedia has shown that simple texts use simpler words, fewer overall words and words that are more general (Coster \& Kauchak, 2011; Napoles \& Dredze, 2010; Zhu, Bernhard, \& Gurevych, 2010). Similar findings have been found in other general domain corpora, such as simplified news texts (Xu, CallisonBurch, \& Napoles, 2015), and in domain-specific analyses as well, e.g. medical texts (Leroy, Endicott, Mouradi, Kauchak, \& Just, 2012; Leroy \& Endicott, 2011). Certain types of words have also been found to be more prevalent in simpler texts including function words and verbs (Kauchak, Leroy, \& Coster, 2012; Leroy \& Endicott, 2011). Word-level effects have 
also been shown in other languages, e.g. Portuguese (Alu et al., 2008) and Spanish (Bott \& Saggion, 2011).

Motivated by these corpus studies, we created a general measure of word difficulty, term familiarity, which relies on the frequency of occurrence of words on the web using the Google Web corpus (Brants \& Franz, 2006). We found that easy texts used more high frequency words (i.e. more familiar words) while difficult texts used more low frequency words (i.e. less familiar) (Leroy et al., 2012; Leroy \& Endicott, 2011). We created a tool that simplifies texts by suggesting simpler (i.e. more frequent) words for difficult (i.e. less frequent) words identified in the text. A user study found that health-related texts simplified by a medical librarian using this tool were viewed as simpler, were easier to understand and resulted in more learning (Leroy, Endicott, et al., 2013). This paper represents a natural extension of this word-level validation process, instead utilizing grammar frequency for simplification.

\section{The Role of Syntax in Simplification}

The syntax or grammar of a language dictates how words and phrases interact to form sentences. Historically, those writing medical text for patients have been encouraged to avoid problematic syntactic structures, e.g. by writing in active voice (vs. passive) and by avoiding long sentences ("Health.gov Quick Guide to Health Literacy," ; "How to Write Easy-to-Read Health Materials,"). In user studies, grammar has been shown to impact text readability, as measured by the Cloze test (Taylor, 1953), which asks participants to fill in missing words in texts. For example, splitting long sentences has been show to improve Cloze scores (Kandula, Curtis, \& Zeng-Treitler, 2010) and additive and causal connectors were easier to fill in than adversative or sequential connectors (Goldman \& Murray, 1992). It has been suggested that grammatical difficulty is particularly important for L2 learners since they are still trying to learn appropriate grammatical structures for the language (Callan \& Eskenazi, 2007; Clahsen \& Felser, 2006). The challenge lies in defining a useful metric to effectively and efficiently measure the grammatical difficulty of a sentence with demonstrated impact on reader understanding through user studies and not expert observations.

In corpus studies, various syntactic differences have been observed between easy and difficult texts. For example, differences in the frequencies of different parts of speech show that easy texts contain a higher proportion of verbs, function words and adverbs, while difficult texts contain a higher proportion of adjectives and nouns (Kauchak et al., 2012; Leroy et al., 2012; Leroy \& Endicott, 2011, 2012; Leroy, Eryilmaz, \& Laroya, 2006; Leroy, Helmreich, \& Cowie, 2008), as well as longer noun phrases (Napoles \& Dredze, 2010). Furthermore, as suggested by medical writing guides, easy texts do tend to use the active voice over the passive (Leroy, Helmreich, \& Cowie, 2010a, 2010b). Differences in highlevel sentence structures have also been seen, e.g. subject-verb-object versus object-subjectverb ordering (Devlin \& Unthank, 2006). Some initial success has been achieved by automated simplification systems that perform syntactic transformations, e.g. dropping prepositional phrases and infinitives and changing verb tenses (Feblowitz \& Kauchak, 2013; 
Laetitia Brouwers, 2014; Woodsend \& Lapata, 2011; Zhu et al., 2010), though much work is still needed.

In previous work, we conducted a preliminary corpus study of grammar frequency which showed that difficult texts use a wider variety of high-level grammatical structures (Kauchak et al., 2012). However, because of the large number of structural variations possible, no clear indication was found showing specific structures predominantly appearing in either easy or difficult documents. In this work, we propose a much more fine-grained analysis. We propose a measure of text difficulty based on grammatical frequency and show how it can be used to identify sentences with difficult syntactic structures. In particular, the grammatical difficulty of a sentence is measured based on the frequency of occurrence of the top-level parse tree structure of the sentence in a large corpus.

\section{METHODS}

\section{Measuring Grammatical Frequency}

To measure the frequency of different grammatical structures, we used the sentences from English Wikipedia (https://en.wikipedia.org/). Wikipedia is one of the most common resources for consumers for general information (Safran, 2012) and is also a useful for corpus for more specialized topics, i.e., approximately $80 \%$ of online users read healthrelated text on the web (Fox, 2011).. We downloaded all articles from English Wikipedia in June, 2013. We then pre-processed the articles, split them into sentences using the Stanford CoreNLP toolkit (Manning et al., 2014) (5.4 million total sentences) and parsed each using the Berkeley Parser (Petrov, Barrett, Thibaux, \& Klein, 2006).

Figure 1 shows two example sentences from the corpus with the induced parse structure. The $1^{\text {st }}$ level of the parse tree of Figure 1a is $S$, the $2^{\text {nd }}$ level [S [NP VP.]] and the $3^{\text {rd }}$ level [S [NP [DT NN]] [VP [VBZ NP]] [..]], which is highlighted with a green dashed box in the figure. Similarly, the $1^{\text {st }}$ level of the parse tree of Figure $1 \mathrm{~b}$ is $S$, the $2^{\text {nd }}$ level $[S$ [NP VP].]] and the 3rd level [S [NP [DT JJS JJ NN] [VP [VBZ NP, S] [..]].

We use the $3^{\text {rd }}$ level of the parse tree to quantify the grammatical difficulty of the sentence. We chose to focus on the $3^{\text {rd }}$ level since it represents a compromise between generality and specificity. At the $2^{\text {nd }}$ level, structures are more general and therefore more likely to match, however, the granularity is less than at the $3^{\text {rd }}$ level; there are 10 times as many unique $3^{\text {rd }}$ level structures as there are $2^{\text {nd }}$ level structures. For example, the sentences in Figure 1 share the same $2^{\text {nd }}$ level parse tree, but have different $3^{\text {rd }}$ level parse trees. However, if we use a deeper depth than the $3^{\text {rd }}$ level there are too many unique structures, reducing the ability to generalize structures across different sentences. $45 \%$ of sentences in the corpus $(2.47 \mathrm{M})$ have unique $4^{\text {th }}$ level parse tree structures, often because the $4^{\text {th }}$ level regularly includes lexical components. For example, the $4^{\text {th }}$ level of the parse tree in Figure 1a would include the words "the heart is" making it very unlikely to generalize to other sentences.

We calculated the frequency of all possible $3^{\text {rd }}$ level structures found in the 5.4 million sentences from Wikipedia resulting in a mapping from any $3^{\text {rd }}$ level structure to its frequency. For example, the structure in Figure 1a is among the most frequent grammatical 
structures. Even with the same underlying $3^{\text {rd }}$ level structure, sentences may present very differently. Table 1 shows examples for different grammatical structures. Each row shows two sentences that have the same $3^{\text {rd }}$ level structure, but that have varying frequency, ordered from most frequent to least. Because we focus on the high-level structure, the length of the sentences with the same structure also can vary widely.

To remove anomalous data and likely misparses, we ignored any structure that had only been seen once among the 5.4 millions sentences. After filtering, this results in 139,969 unique $3^{\text {rd }}$ level structures. Even with removal of unique grammatical structures, the frequency distribution of sentences is extremely skewed. Figure 2 shows a plot of the log of the frequency of the structures, sorted from most frequent to least. Like many other text phenomena, grammatical frequency follows a Zipf -like distribution, with the most common structures occurring very frequently and many structures occurring infrequently, though grammar frequency is particularly extreme.

\section{Applying the Grammatical Frequency Measure}

Given any sentence, the grammatical frequency can be calculated by:

1. parsing the sentence using the Berkeley parser,

2. extracting the $3^{\text {rd }}$ level parse tree and

3. looking up the frequency of that structure in the grammar frequency database.

Structures that are not found can be given a frequency of 0 . We hypothesize that sentences with structures that are more frequent are easier to comprehend and those that are less frequent are more difficult to comprehend. This approach for measuring the grammatical difficulty of text represents a generalized and data-driven approach that goes beyond specific, theory-based grammatical components of text difficult (e.g. active vs. passive voice, self-embedded clauses, etc. (Meyer \& Rice, 1984)) and provides a generic framework for measuring grammatical difficulty. Individual structures, such as passive vs. active, could be evaluated as a subset of our general approach.

\section{Evaluation of the Measure's Ability to Estimate Sentence Difficulty: User Study}

To evaluate grammatical frequency and its relation to reader comprehension, we conducted a user study with the main variable of interest the grammatical frequency of the sentence. To minimize confounding factors that might influence sentence difficulty we control for sentence length and term familiarity.

Stimuli-We ranked the 139,939 unique $3^{\text {rd }}$ level structures and divided them into 11 frequency bins. The $1^{\text {st }}$ bin contained the top $1 \%$ most frequently used grammatical structures. The next $10 \%$ most frequent went into the $2^{\text {nd }}$ bin, the next $10 \%$ into the $3^{\text {rd }}$ bin, etc. Finally, the $11^{\text {th }}$ bin contains the remaining sentences with the $10 \%$ least frequent grammatical structures. While different numbers of bins can be tested, we have chosen eleven bins to see the effect of the most frequent structures (top bin) and ten additional bins to see trends if they exist. 
Each of the 5.4 million Wikipedia sentences can be mapped to one of the 11 frequency bins and we selected a subset of these for our study. To avoid very long and very short sentences, and to partially mitigate length effects, we only sampled from sentences that had a length within one standard deviation from the average sentence length of the corpus. Assuming the lengths follow a normal distribution, this samples from roughly $2 / 3^{\text {rds }}$ of the sentences, removing the $1 / 6^{\text {th }}$ shortest and $1 / 6^{\text {th }}$ longest sentences.

For each of the 11 frequency bins, we randomly selected 20 sentences. To control for other text characteristics that might impact text difficulty and to see how grammar frequency interacts with secondary characteristics we controlled for two variables when selecting these sentences: sentence length and term familiarity, both of which have been previously shown to impact text difficulty (Kim et al., 2007; Kincaid et al., 1975; Leroy et al., 2012; Leroy \& Kauchak, 2013; McLaughlin, 1969).

To control for sentence length, sentences in the remaining corpus were divided into long, average and short sentences, representing the top third, middle third and bottom third by length, respectively. In each frequency bin, we then selected half (ten) long sentences and half short sentences. To control for term familiarity, we calculated the term familiarity of each sentence as the average term familiarity of each word in that sentence. The familiarity of each word was measured as the frequency in the Google Web Corpus, roughly approximating the frequency of occurrence on the web. Like length, we divided sentences into thirds based on average term familiarity and each bin included half (ten) from the top third ("high" familiarity) and half from the bottom third ("low" familiarity).

This process resulted in a sample of 220 sentences in 11 frequency bins with each bin containing 5 long sentences with high familiarity, 5 long with low familiarity, 5 short with high familiarity, and 5 short with low familiarity. Table 2 shows example sentences for each these four categories from three of the bins.

Table 3 shows summary statistics for the 220 sentences used in the study, averaged over the frequency bins. The sentence lengths and term familiarity were roughly equal across all bins. On average, the long sentences were 1.8 times longer (contained 14 more words) and the sentences with higher frequency words contained words that were on average 1.7 times more frequent. A paired-samples $t$-test showed our two control variables to be effective, with length significantly different between short and long sentences $(t(10)=-60.47, p<0.001)$ and word frequency significantly different between the high and low group $(\mathrm{t}(10)=-38.47$, $\mathrm{p}<0.001)$. The impact of grammar frequency, our variable of interest, is discussed in the results section.

Metrics for Evaluating Text Difficulty-To evaluate how difficult a sentence is, we rely on evidence of comprehension by laypersons. We do not rely on expert judgments or readability formulas. Following our previous work (Leroy et al., 2012; Leroy, Endicott, et al., 2013), we decompose text difficulty into two separate components: actual difficulty and perceived difficulty. Actual difficulty measures how well people comprehend a particular text, while perceived difficulty measures how difficult people perceive a text to be. Actual difficulty has been measured with a variety of metrics including multiple choice questions, 
problem solving questions, inference questions (McNamara, Kintsch, Songer, \& Kintsch, 1996), and the Cloze measure (Taylor, 1953). Separating text difficulty into perceived and actual difficulty is important since both can play a role in whether or not a reader will obtain the required information from a text (Janz \& Becker, 1984; Leroy, Kauchak, \& Mouradi, 2013).

To measure actual difficulty (first dependent variable) we used a Cloze test. The basic Cloze test involves replacing every $n^{\text {th }}$ word in a text with a blank. Participants are then asked to fill in the blanks and are scored based on how many of their answers matched the original text (Taylor, 1953). The Cloze test was originally intended to show differences in difficulty between different texts, though it has also been used to measure the readability levels of texts (Collins, Currie, Bakken, Vawdrey, \& Stone, 2012; Taylor, 1953) and user comprehension (Bormuth, 1969; Siddharthan, 2002). It has been used in many studies including measuring $2^{\text {nd }}$ language proficiency (Goldman \& Murray, 1992), the impact of reading habits on language proficiency (Leroy, Kauchak, et al., 2013) and to evaluate simplification procedures (Kandula et al., 2010).

We employed a multiple-choice Cloze test. For each sentence, four nouns were randomly selected and replaced with blanks. For each sentence, we create five multiple-choice options containing the four removed words in different random orders, one of which is the correct ordering. Using the multiple-choice version of the Cloze test allows for automated scoring and removes any subjectiveness during evaluation. Figure 3 shows an example blanked sentence and its five options (option $\mathbf{b}$ is the correct answer). We only allowed sentence/ noun selections where the ordering of nouns was unambiguous, e.g. a comma separated list of nouns would not be allowed, since any ordering of the nouns would be valid.

To measure perceived difficulty (second dependent variable), participants were asked to rate the sentences on a 5-point Likert scale with higher numbers representing more difficult sentences. Specifically, we posed the question "How difficult would this sentence look in a text" and presented five options: Very Easy, Easy, Neither, Difficult, and Very Difficult.

In addition to perceived and actual difficulty, we also collected the time that participants took to complete the task (third dependent variable). Completion time is related to reading time, which has been frequently used as a measure of cognitive load (Raney, 1993) and text difficulty (Cirilo \& Foss, 1980; Mandler \& Goodman, 1982).

Study Participants-We recruited participants for the study from Amazon Mechanical Turk (MTurk). MTurk is a crowdsourcing tool where requesters can upload tasks to be accomplished by a set of workers for a fee. MTurk has been used in many research settings ranging from user studies to data annotation to subjective rating generation (Hao, Rusanov, Boland, \& Weng, 2014; Kittur, Chi, \& Suh, 2008) and has been shown to provide results similar to those from other human subjects. In addition, it enables the use of a large population of participants (over half a million) from a diverse group with varied demographic characteristics (Paolacci, Chandler, \& Ipeirotis, 2010; Ross, Irani, Silberman, Zaldivar, \& Tomlinson, 2010). 
For each of the 220 sentences, we recruited 30 participants for a total of $\mathrm{N}=6,600$ samples. To ensure the quality and accuracy of the data, participants were restricted to be within the United States and to have a previous approval rating of 95\%. Because of the setup of MTurk, participants can choose how many sentences to complete. MTurk then ensures that for a given sentence, each of the 30 participants is different. For all participants we collected demographic data including gender, age, education level, ethnicity and race.

\section{RESULTS}

\section{Participants}

A total of 143 people participated in the study. Participants completed 46 sentences on average, though the median was lower at 18 sentences. Table 4 shows participant demographic information. There were roughly an equal number of male and female participants. $50 \%$ of the participants were under 30 and $90 \%$ under 50 . Every participant had at least a high school diploma and $43 \%$ had at least a bachelor's degree. The participants were predominantly white $(87 \%)$.

\section{Actual Difficulty}

We analyzed the impact of grammar frequency (main variable of interest) as well as sentence length and term familiarity (two control variables) on actual difficulty. We conducted an ANOVA with three independent variables: grammar frequency bin (1-11 bins), sentence length (long or short) and average word frequency (high or low). Each condition $(11 \times 2 \times 2)$ had 5 sentences and for each sentence we gathered 30 responses, resulting in a dataset of $\mathrm{N}=6,600$. The ANOVA allows us to determine whether there are any main effects of any of our variables, i.e., an effect of a variable across all levels of another variable, and interaction effects, i.e. an effect of a variable that varies depending on the level of another variable, between our variables of interest (grammar frequency, sentence length and term familiarity) for our evaluation metric (in this case, actual difficulty. It does this with a single statistical test, avoiding the problem of compounding uncertainty when applying multiple tests, e.g. t-tests.

Figure 4a shows the actual difficulty scores averaged over the frequency bins. Since the multiple-choice Cloze test provides participants with multiple clues to select the correct answer, overall the scores tend to be higher. For more frequent structures the accuracy on the Cloze test is fairly consistent, around $90 \%$ correct. However, as the grammatical structures become less frequent the accuracy drops fairly consistently to a final rate of around $86 \%$ (starting at bin 6). The ANOVA shows this effect to be significant $(\mathrm{F}(10,6556)=5.404, \mathrm{p}<$ $0.001)$, i.e. there is an effect of grammar frequency on actual difficulty. There were no main effects of sentence length or term familiarity.

Our results showed interaction effects. The impact of grammar frequency is much less pronounced for short sentences (Figure $4 \mathrm{~b}$ ) and for sentences with lower frequency words (Figure 4c). An ANOVA shows these differences to be significant $(\mathrm{F}(10,6556)=3.453$, $\mathrm{p}<$ $0.001)$, for grammar frequency and sentence length, and $(F(10,6556)=1.870, p=0.044)$, for 
grammar frequency and term familiarity. In addition, the interaction between all three variables is also significant $(\mathrm{F}(10,6556)=4.650, \mathrm{p}<0.001)$.

To complete this analysis and understand the strength of the effect on actual difficulty, we calculated a one-tailed Pearson correlation coefficient between the grammar frequency and the actual difficulty (percentage correct) for both the raw scores and scores aggregated by frequency bin. There was a negative correlation between grammar frequency and the actual difficulty of the sentence (raw scores: $\mathrm{N}=6,600, \mathrm{r}=-0.053$, $\mathrm{p}<0.01$; bin averages: $\mathrm{N}=11$, $\mathrm{r}=-0.596, \mathrm{p}<0.05)$ indicating that sentences that used less frequent structures were harder to understand.

\section{Perceived difficulty}

We conducted a parallel analysis for perceived difficulty. Figure 5 shows the average perceived difficulty rating of the 220 sentences by the participants per bin. Following common practice, we treat this scale as continuous and calculate average scores per bin (Norman, 2010). Lower scores indicate sentences that are perceived to be easier. For more frequent structures, the difficulty rating oscillates around 2.0, however, as the structures become less familiar (decreasing in frequency) they are perceived to be more difficult. An ANOVA for perceived difficulty shows a main effect for frequency bin $(\mathrm{F}(10,6556)=9.108$, $\mathrm{p}<0.001)$. In contrast to actual difficulty, we also find a main effect of the sentence length on perceived difficulty with longer sentences seen as more difficult (averaged 2.2) than the shorter sentences (averaged 2.0). Surprisingly, there was no effect of the average term frequency on perceived difficulty.

As with actual difficulty, several interaction effects are significant and can be seen in Figure $5 b$ and $5 c$. The effect of grammar frequency on perceived difficulty is smaller in shorter sentences and those with lower term frequency. An ANOVA shows both these interaction to be significant $(F(10,6556)=5.648, p<0.001$, for grammar frequency and sentence length, and $F(10,6556)=5.332, p<0.001$, for grammar frequency and term familiarity). Both high and low frequency sentences show a jump in difficulty, though it occurs earlier (bin 7) for low frequency sentences than for high frequency sentences (bin 8).

Although not our main interest, we also report a significant interaction between the sentence length and term familiarity on perceived difficulty $(\mathrm{F}(10,6556)=4.252, \mathrm{p}=0.039)$ and a significant three-way interaction between all three variables $(F(10,6556)=5.734, p<0.001)$.

We complete our analysis with a one-tailed Pearson correlation coefficient between the grammar frequency and the perceived difficulty. There is a positive correlation between frequency and perceived difficulty: participants judged sentences with less frequent grammatical structures as more difficult $(\mathrm{N}=6600, \mathrm{r}=0.042, \mathrm{p}<0.01)$.

Finally, comparing both our dependent variables (actual and perceived difficulty), we found a significant correlation between how well readers performed on the Cloze test and how difficult they thought a sentence was. Lower accuracy correlated with higher difficulty scores $(\mathrm{N}=11, \mathrm{r}=-0.574, \mathrm{p}<0.05 ; \mathrm{N}=6600, \mathrm{r}=-0.203, \mathrm{p}<0.01)$ : sentences that were harder for participants to understand also looked more difficult. This reiterates the need to 
make sure that sentences are both more understandable (actual difficulty) as well as seen as simpler (perceived difficulty).

Time

We conducted an ANOVA on the total time taken to complete the task per sentence, which includes the time to read the sentence, perform the Cloze test and perform the subjective difficulty judgment. However, since perceived difficulty is always measured with the same 5-point Likert scale, we posit that the time taken mainly reflects the time needed to read the sentence and solve the Cloze task. We found a main effect of grammar frequency on time, with sentences in lower frequency bins taking more time $(F(10,6556)=3.575, p<0.001)$. In addition, there was the expected main effect of sentence length, with longer sentences taking more time. No other main or two-way interactions were significant, however, the three way interaction between all three independent variables was significant $(\mathrm{F}(10,6556)=1.996, \mathrm{p}=$ $0.030)$.

Correlation analysis confirms these results and provides a sense of the strength of the relationship. There was a significant correlation between the grammar frequency and the time spent $(\mathrm{N}=6600, \mathrm{r}=0.024, \mathrm{p}<0.05)$ : sentences with less familiar grammatical structures took longer to accomplish the task. Recall that the sentences in the bins have equivalent lengths and term familiarity, two other factors that could play a role in the time spent.

Both perceived and actual difficulty correlated as expected with the time spent: participants spent longer on sentences that they thought were more difficult $(\mathrm{N}=11, \mathrm{r}=0.936, \mathrm{p}<0.01$; $\mathrm{N}=6600, \mathrm{r}=0.208, \mathrm{p}<0.01$ ) and they also spent more time on sentences that were actually more difficult $(\mathrm{N}=11, \mathrm{r}=-0.538, \mathrm{p}<0.05 ; \mathrm{N}=6600, \mathrm{r}=-0.093, \mathrm{p}<0.01)$.

\section{Summary of Effects}

Table 5 shows a summary of all of the ANOVA results for the three evaluation metrics.

\section{Relationship to Existing Difficulty Measures}

Readability formulas are the main tool used in the medical community to evaluate text difficulty and to "guide" text simplification (Weiss, 2007). To evaluate the effectiveness of these readability formulas for predicting text difficulty, we calculated readability scores for the 220 sentences using three of the most commonly used readability metrics: Flesch reading ease score, Flesch-Kincaid (FK) grade level (Kincaid et al., 1975) and SMOG readability formula (McLaughlin, 1969).

We calculated a two-tailed Pearson correlation coefficient between each of the readability measures and the dependent variables used to measure text in our user study (perceived difficulty, actual difficulty and time). None of the three readability metrics correlated significantly with either actual difficulty or time. The readability metrics that are currently used do not predict how likely users are to understand the content of the text.

All three readability metrics did, however, correlate significantly with perceived difficulty (Flesch: $\mathrm{r}=-0.22, \mathrm{p}<0.001$; FK: $\mathrm{r}=0.25, \mathrm{p}<0.001$; SMOG: $\mathrm{r}=0.10, \mathrm{p}<0.01$ ): sentences 
that were scored as easier by the readability metrics were seen as easier by people. While this may initially seem beneficial, this too can be problematic when employed for simplifying text. These readability metrics are used to guide medical writers in creating more understandable text. Writers may create text that they perceive to be simpler and validate this with the readability formulas, however, they may not be creating text that is actually easier for readers understand to understand.

As an example, Figure 6 shows the actual difficulty and perceived difficulty for the sentences relative to the Flesch-Kincaid grade level. As the correlation results indicate, there is an increase in the perceived difficulty as the grade level increases. However, the actual difficulty remains more or less constant as the grade level increases, i.e. even though FleschKincaid indicates that the text is getting more difficult, participants understood the text equally well. The noisier results at small and large grade-levels are due to the small number of sentences that had this grade level.

\section{GRAMMAR FAMILIARITY AS AN ANALYSIS TOOL}

Grammar frequency can also be used as a corpus analysis tool to understand the high-level grammatical characteristics of a corpus.

\section{Corpora}

As a demonstration study, we collected three different medical corpora discussing common diseases. We selected diseases from the leading causes of death from the CDC's most recent mortality study (Murphy, Kochanek, Xu, \& Heron, 2015): heart disease, cancer, respiratory disease, stroke, Alzheimer's disease, diabetes, influenza, pneumonia, nephritis, nephrosis and suicide. The corpora were collected from different sources patients might turn to for health information about these diseases:

- $\quad$ PubMed: We searched the PubMed database, which contains abstracts from medical-related research publications, for each of the above diseases and downloaded all abstracts from the resulting matched papers.

- Cochrane: The Cochrane database (http://www.cochrane.org/) contains survey reviews of health care and health policy research and can be a useful research source for patients and practitioners to find out about current medical research on a topic. Similar to PubMed, we searched for each of the above diseases and downloaded all article abstracts for the matching reviews.

- Blogs: We identified ten different blogs discussing common medical diseases and downloaded all posts from the sites. The blogs were not written by medical researchers or medical professionals.

Each of the texts were tokenized and split into sentences using the Stanford CoreNLP toolkit and then parsed using the Berkeley Parser (the same preprocessing as the frequency bins). Table 6 shows the number of articles and the number of sentences for each of the three corpora. 
For each sentence we determined which grammar familiarity bin the sentence belonged to from the $3^{\text {rd }}$ level of the parse tree. $3^{\text {rd }}$ level structures that were not found (i.e. had not been previously seen in the database) were put into the last $\left(11^{\text {th }}\right)$ bin since they represent very infrequent structures (i.e. frequency 0 in the database).

\section{Results}

Figure 7 shows the proportion of sentences from each of the three corpora that fell into each of the 11 grammar bins. Several key findings stand out. All corpora followed more or less the expected pattern with most of the sentences falling into the most frequent bin and then decreasing numbers in the subsequent bins. PubMed had the most sentences in bin 1 (the most frequent bin) of the three corpora. Looking at the sentences in bin 1, many of these can be attributed to the consistent writing style used in writing abstracts for health-related research. Cochrane had almost $10 \%$ of the sentences fall into bin 8 , which at first glance is surprising. However, this came again from a common pattern used in Cochrane abstracts to highlight different aspects of the reviews, e.g. "Main result: ..." and "Selection criteria: ...". All three corpora also had many sentence structures that were not previously seen, resulting in a large number in bin 11. Since blogs tended to use a more casual (and sometimes ungrammatical) writing style, blogs had the most in this category.

For these three corpora, we already posses a high-level understanding of the writing style (i.e. research abstracts tend to be technical, but use common structures and blogs tend to use more varied and more casual grammar structures). For new corpora, grammar familiarity can help understand the regularity of the grammar structures used. Additionally, even for known corpora, it can help identify other regularities/anomalies not previously known, for example in the Cochrane corpus.

This study also highlights that grammar frequency is just one component of text difficulty. To avoid confounding factors, in the user study, we kept term familiarity (as a proxy for word difficulty) constant across the different grammatical structures. Without doing this, it can be harder to measure the impact of secondary difficulty characteristics like grammar. For example, in the analysis above PubMed abstracts are generally more difficult to understand than blogs, but based only on grammar frequency they appear easier, containing more frequent structures. This analysis, though, ignores word difficulty. The PubMed corpus used more infrequent terms with an average term familiarity of $2.83 \mathrm{M} /$ word compared to the blogs corpus of $2.96 \mathrm{M} /$ word.

\section{DISCUSSION}

In this study we provide two main contributions. First, we show that current readability metrics do not effectively measure text difficulty. Readability measures based on word and sentence length correlate with how difficult people perceive sentences to be but they do not correlate with how likely a person is to understand that sentence. Second, and more importantly, we introduce a new measure of text difficulty that utilizes the frequency of occurrence of the $3^{\text {rd }}$ level grammatical structure, where frequency is calculated using structures from all sentences in English Wikipedia. This metric can be applied to new sentences to predict sentence difficulty. We validated this new measure of text difficulty with 
a user study examining 220 sentences with 30 evaluations per sentence $(\mathrm{N}=6,600)$ and found that there is a significant effect of grammar frequency on both how difficult a person thinks that sentence is (perceived difficulty) as well as how easy that sentence is to understand (actual difficulty).

Not surprisingly, actual difficulty is less affected by grammar in short sentences. One explanation may be that shorter sentences are easy to understand and any effect of grammar is difficult to detect (ceiling effect). Similarly, in sentences with low term familiarity (i.e. more difficult words) the grammar familiarity doesn't impact text difficulty since users are struggling with the lexical difficulty. However, in sentences with very familiar terms, which are easier to understand, grammar frequency does have an impact on actual difficulty; only in sentences where the words are more familiar does the grammatical frequency have a strong effect. Interestingly, there was very little impact overall of term frequency on actual difficulty. Based on these observations, we hypothesize that there is a relation between grammatical frequency and term frequency. Future studies are required to fully validate these hypotheses.

Our study has limitations. Text comprehension was measured with individual sentences. Working with longer text may reduce the effects when more contextual information is available. Our statistical analysis shows significant interactions only and we used visual inspection to interpret the different patterns. Further investigation is required to validate these patterns.

Given a new sentence, the grammar frequency can be used to estimate the grammatical difficulty of the sentence automatically, as illustrated in the corpus analysis. We have generated a preliminary tool that does this by parsing the sentence, extracting the $3^{\text {rd }}$ level parse tree and then looking up the corresponding grammar frequency bin. Sentences that have problematic (i.e. low frequency structures) can be flagged automatically for the medical writer to then correct to an easier variant (i.e. higher frequency structure). For example, even a simple heuristic of identifying all sentences in bins 7-11 would flag sentences with significantly lower user comprehension. In our study, comparing the sentences from bins 711 to those in bins 1-6: perceived difficulty is lower 2.26 vs 2.0 , a $12 \%$ reduction; actual difficulty is $87.6 \%$ vs. $91.4 \%$, a difference of $\sim 4 \%$; and the time spent to process the sentence is $39.8 \mathrm{~s}$ vs. $44.3 \mathrm{~s}$, a $4.5 \mathrm{~s}$ increase. The optimal number and distribution of bins can be tested statistical by evaluating different permutations. However, the number of bins also has implications for our future tool. Smaller bins represent higher granularity and will allow for more fine-tuned feedback and suggestions, but require more data to get good estimates of the bin quality. Larger bins are easier to collect data for, but represent lower granularity in grammar differences and will require tool users to make larger changes in the grammar without tool feedback. In future work, we plan to further enhance the current tool by suggesting transformation that improve the grammatical frequency, e.g. by learning grammatical transformation rules from a parallel corpus of normal and simplified sentences (Feblowitz \& Kauchak, 2013; Woodsend \& Lapata, 2011; Zhu et al., 2010). 


\section{Acknowledgments}

Research reported in this publication was supported by the National Library Of Medicine of the National Institutes of Health under Award Number R01LM011975. The content is solely the responsibility of the authors and does not necessarily represent the official views of the National Institutes of Health.

\section{REFERENCES}

Adkins AD, Singh NN. Reading level and readability of patient education materials in mental health. Journal of Child and Family Studies. 2001; 10(1):1-8.

Alu, SM., Specia, L., Pardo, TAS., Maziero, EG., Fortes, RPM. Towards Brazilian Portuguese automatic text simplification systems; Paper presented at the Proceedings of the eighth ACM symposium on Document engineering; Sao Paulo, Brazil. 2008. \#237, sio

Bailin A, Grafstein A. The linguistic assumptions underlying readability formulae: A critique. Language \& Communication. 2001; 21(3):285-301.

Bormuth JR. Factor Validity of Cloze Tests as Measures of Reading Comprehension Ability. Reading Research Quarterly. 1969; 4(3):358-365.

Bott S, Saggion H. Spanish text simplification: An exploratory study. Procesamiento del lenguaje natural. 2011; 47:87-95.

Brainard J. Study finds research consent forms difficult to comprehend. The Chronicle of higher education. 2003; 49(19):A21-A22. [PubMed: 15287115]

Brandt HM, McCree DH, Lindley LL, Sharpe PA, Hutto BE. An evaluation of printed HPV educational materials. Cancer Control. 2005; 12(suppl 2):103-106. [PubMed: 16327760]

Brants T, Franz A. The Google Web 1T 5-gram corpus version 1.1. 2006 LDC2006T13.

Bruce BC, Rubin A, Starr KS. Why readability formulas fail. 1981

Callan, J., Eskenazi, M. Combining lexical and grammatical features to improve readability measures for first and second language texts; Paper presented at the NAACL HLT; 2007.

Cirilo RK, Foss DJ. Text structure and reading time for sentences. Journal of Verbal Learning and Verbal Behavior. 1980; 19(1):96-109.

Clahsen H, Felser C. Grammatical processing in language learners. Applied Psycholinguistics. 2006; 27(01):3-42.

Collins SA, Currie LM, Bakken S, Vawdrey DK, Stone PW. Health literacy screening instruments for eHealth applications: a systematic review. Journal of biomedical informatics. 2012; 45(3):598607. [PubMed: 22521719]

Collins-Thompson K, Callan J. Predicting reading difficulty with statistical language models. Journal of the American Society for Information Science and Technology. 2005; 56(13):1448-1462.

Committee on Health Literacy - Institute of Medicine of the National Academies. 2004 Retrieved from https://iom.nationalacademies.org/ /media/Files/ReportFiles/2004/Health-Literacy-APrescription-to-End-Confusion/healthliteracyfinal.pdf.

Connatser BR. Last rites for readability formulas in technical communication. Journal of technical writing and communication. 1999; 29(3):271-288.

Coster, W., Kauchak, D. Simple English Wikipedia: a new text simplification task; Paper presented at the Proceedings of the 49th Annual Meeting of the Association for Computational Linguistics: Human Language Technologies: short papers - Volume 2; Portland, Oregon. 2011.

Devlin, S., Unthank, G. Helping aphasic people process online information; Paper presented at the Proceedings of the 8th international ACM SIGACCESS conference on Computers and accessibility; Portland, Oregon, USA. 2006.

Farmer AP, Légaré F, Turcot L, Grimshaw J, Harvey E, McGowan JL, Wolf F. Printed educational materials: effects on professional practice and health care outcomes. Cochrane Database Syst Rev. 2008; 3(3)

Feblowitz, D., Kauchak, D. Sentence simplification as tree transduction; Paper presented at the Proc. of the Second Workshop on Predicting and Improving Text Readability for Target Reader Populations; 2013. 
Fox S. Health topics. Pew Internet \& American Life Project. 2011

Goldman SR, Murray JD. Knowledge of connectors as cohesion devices in text: A comparative study of native-English and English-as-a-second-language speakers. Journal of Educational Psychology. 1992; 84(4):504.

Hao T, Rusanov A, Boland MR, Weng C. Clustering clinical trials with similar eligibility criteria features. Journal of biomedical informatics. 2014; 52:112-120. [PubMed: 24496068]

Health.gov Quick Guide to Health Literacy. Retrieved from http://www.health.gov/communication/ literacy/quickguide/factsbasic.htm

How to Write Easy-to-Read Health Materials. Retrieved from http://www.nlm.nih.gov/medlineplus/ etr.html

Janz NK, Becker MH. The health belief model: A decade later. Health Education \& Behavior. 1984; 11(1):1-47.

Kandula, S., Curtis, D., Zeng-Treitler, Q. A semantic and syntactic text simplification tool for health content; Paper presented at the AMIA Annu Symp Proc; 2010.

Kauchak, D., Leroy, G., Coster, W. A Systematic Grammatical Analysis of Easy and Difficult Medical Text; Paper presented at the American Medical Infomatics Association (AMIA) Fall Symposium; 2012.

Kim, H., Goryachev, S., Rosemblat, G., Browne, A., Keselman, A., Zeng-Treitler, Q. Beyond surface characteristics: a new health text-specific readability measurement; Paper presented at the AMIA Annual Symposium Proceedings; 2007.

Kincaid JP, Fishburne Jr RP, Rogers RL, Chissom BS. Derivation of new readability formulas (automated readability index, fog count and flesch reading ease formula) for navy enlisted personnel. 1975 Retrieved from.

Kittur, A., Chi, EH., Suh, B. Crowdsourcing user studies with Mechanical Turk; Paper presented at the Proceedings of the SIGCHI conference on human factors in computing systems; 2008.

Laetitia Brouwers, DB., Anne-Laure, Ligozat, Thomas, Francois. Syntactic Sentence Simplification for French; Paper presented at the Proceedings of the 3rd Workshop on Predicting and Improving Text Readability for Target Reader Populations (PITR)@ EACL; 2014.

Leroy, G., Endicott, J., Mouradi, O., Kauchak, D., Just, M. Improving perceived and actual text difficulty for health information consumers using semi-automated methods; Paper presented at the AMIA Fall Symposium; 2012.

Leroy, G., Endicott, JE. Digital Libraries: For Cultural Heritage, Knowledge Dissemination, and Future Creation. Springer; 2011. Term familiarity to indicate perceived and actual difficulty of text in medical digital libraries; p. 307-310.

Leroy, G., Endicott, JE. Combining NLP with evidence-based methods to find text metrics related to perceived and actual text difficulty; Paper presented at the Proceedings of the 2nd ACM SIGHIT International Health Informatics Symposium; 2012.

Leroy G, Endicott JE, Kauchak D, Mouradi O, Just M. User evaluation of the effects of a text simplification algorithm using term familiarity on perception, understanding, learning, and information retention. Journal of medical Internet research. 2013; 15(7)

Leroy, G., Eryilmaz, E., Laroya, BT. Health information text characteristics; Paper presented at the AMIA Annual Symposium; 2006.

Leroy G, Helmreich S, Cowie J. Evaluating online health information: Beyond readability formulas. 2008

Leroy, G., Helmreich, S., Cowie, JR. The effects of linguistic features and evaluation perspective on perceived difficulty of medical text; Paper presented at the System Sciences (HICSS), 2010 43rd Hawaii International Conference on; 2010a.

Leroy G, Helmreich S, Cowie JR. The influence of text characteristics on perceived and actual difficulty of health information. International journal of medical informatics. 2010b; 79(6):438449. [PubMed: 20202895]

Leroy G, Kauchak D. The effect of word familiarity on actual and perceived text difficulty. Journal of the American Medical Informatics Association. 2013 amiajnl-2013-002172. 
Leroy G, Kauchak D, Mouradi O. A user-study measuring the effects of lexical simplification and coherence enhancement on perceived and actual text difficulty. International journal of medical informatics. 2013; 82(8):717-730. [PubMed: 23639262]

Leroy G, Miller T, Rosemblat G, Browne A. A balanced approach to health information evaluation: A vocabulary-based naïve Bayes classifier and readability formulas. Journal of the American Society for Information Science and Technology. 2008; 59(9):1409-1419.

Mandler JM, Goodman MS. On the psychological validity of story structure. Journal of Verbal Learning and Verbal Behavior. 1982; 21(5):507-523.

Manning, C., Surdeanu, M., Bauer, J., Finkel, J., Bethard, SJ., McClosky, D. The Stanford CoreNLP Natural Language Processing Toolkit; Paper presented at the Association for Computational Linguistics: System Demonstrastions; 2014.

Maples P, Franks A, Stevens AB, Wallace LS. Development and validation of a low-literacy Chronic Obstructive Pulmonary Disease knowledge Questionnaire (COPD-Q). Patient education and Counseling. 2010; 81(1):19-22. [PubMed: 20044232]

McLaughlin GH. SMOG grading: A new readability formula. Journal of reading. 1969; 12(8):639646.

McNamara DS, Kintsch E, Songer NB, Kintsch W. Are good texts always better? Interactions of text coherence, background knowledge, and levels of understanding in learning from text. Cognition and instruction. 1996; 14(1):1-43.

Meyer BJ, Rice GE. The structure of text. Handbook of reading research. 1984; 1:319-351.

Murphy SL, Kochanek KD, Xu J, Heron M. National vital statistics reports. National Vital Statistics Reports. 2015; 63(9)

Napoles, C., Dredze, M. Learning simple Wikipedia: a cogitation in ascertaining abecedarian language; Paper presented at the Proceedings of the NAACL HLT 2010 Workshop on Computational Linguistics and Writing: Writing Processes and Authoring Aids; Los Angeles, California. 2010.

Norman G. Likert scales, levels of measurement and the "laws" of statistics. Advances in health sciences education. 2010; 15(5):625-632. [PubMed: 20146096]

Paolacci G, Chandler J, Ipeirotis PG. Running experiments on amazon mechanical turk. Judgment and Decision making. 2010; 5(5):411-419.

Petrov, S., Barrett, L., Thibaux, R., Klein, D. Learning accurate, compact, and interpretable tree annotation; Paper presented at the Proceedings of the 21st International Conference on Computational Linguistics and the 44th annual meeting of the Association for Computational Linguistics; Sydney, Australia. 2006.

Pitler, E., Nenkova, A. Revisiting readability: A unified framework for predicting text quality; Paper presented at the Proceedings of the Conference on Empirical Methods in Natural Language Processing; 2008.

Raney GE. Monitoring changes in cognitive load during reading: an event-related brain potential and reaction time analysis. Journal of Experimental Psychology: Learning, Memory, and Cognition. 1993; 19(1):51.

Rathbun A, Thornton LA, Fox JE. Are Our Investments Paying Off?: A Study of Reading Level and Bereavement Materials. American Journal of Hospice and Palliative Medicine. 2008; 25(4):278281. [PubMed: 18463410]

Ross, J., Irani, L., Silberman, M., Zaldivar, A., Tomlinson, B. Who are the crowdworkers?: shifting demographics in mechanical turk; Paper presented at the CHI'10 Extended Abstracts on Human Factors in Computing Systems; 2010.

Safran N. Wikipedia in the SERPs. 2012 Retrieved from http://www.conductor.com/blog/2012/03/ wikipedia-in-the-serps-appears-on-page-1-for-60-of-informational-34-transactional-queries/.

Siddharthan, A. An architecture for a text simplification system; Paper presented at the Language Engineering Conference; 2002; 2002. Proceedings

Taylor WL. "Cloze procedure": a new tool for measuring readability. Journalism Quarterly. 1953; 30:415-433.

Vernon JA, Trujillo A, Rosenbaum SJ, DeBuono B. Low health literacy: Implications for national health policy. 2007 
Weiss BD. Health literacy and patient safety: Help patients understand (Manual for clinicians). 2007

Woodsend, K., Lapata, M. Learning to simplify sentences with quasi-synchronous grammar and integer programming; Paper presented at the Proceedings of the conference on empirical methods in natural language processing; 2011.

Xu W, Callison-Burch C, Napoles C. Problems in current text simplification research: New data can help. Transactions of the Association for Computational Linguistics. 2015; 3:283-297.

Zhu, Z., Bernhard, D., Gurevych, I. A monolingual tree-based translation model for sentence simplification; Paper presented at the Proceedings of the 23rd international conference on computational linguistics; 2010. 


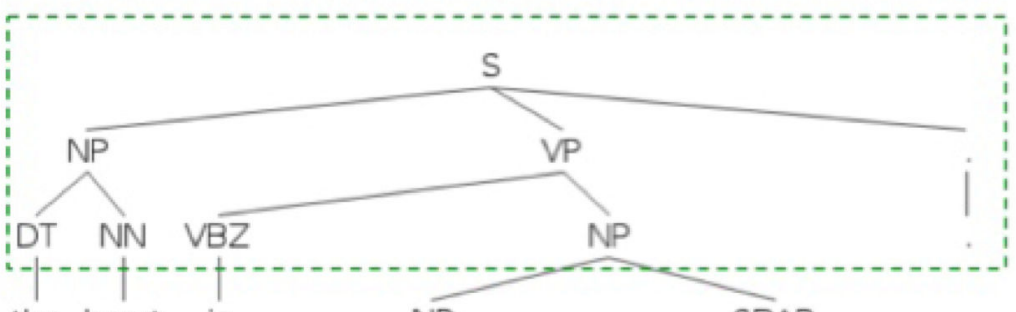

the heart is
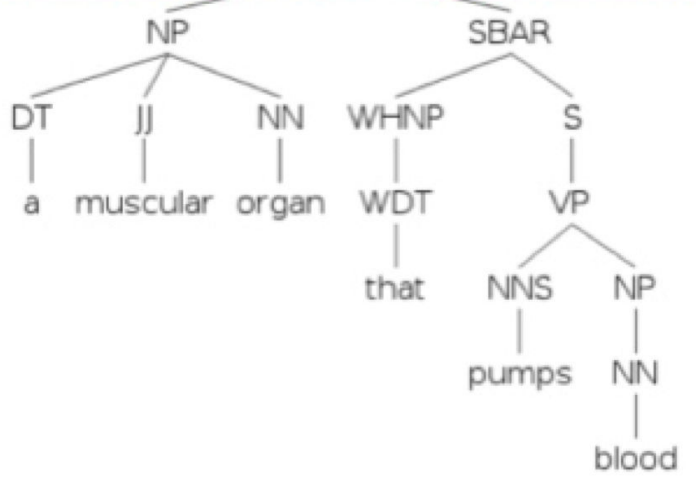

(a)

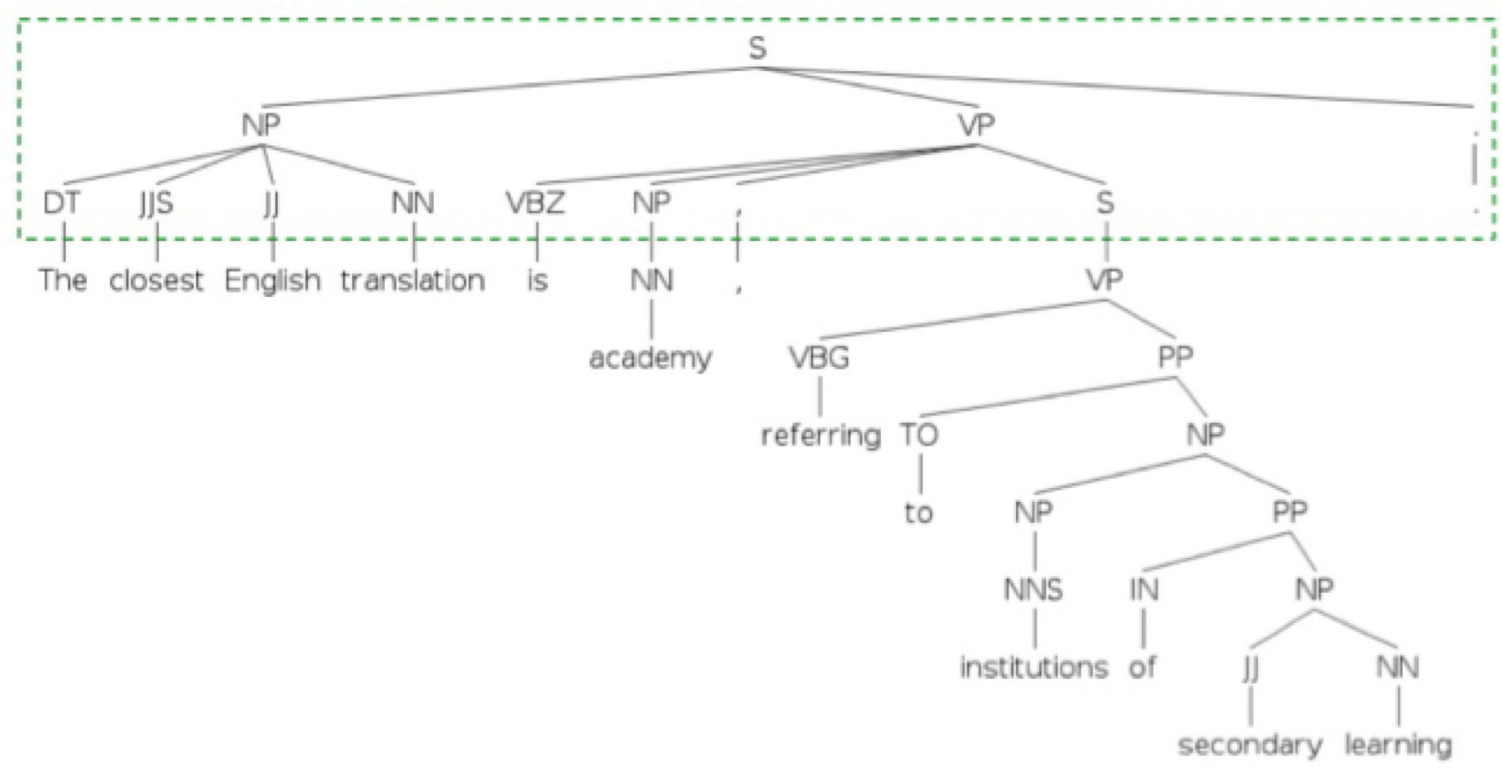

(b)

Figure 1.

Example parse trees generated automatically using the Berkeley parser. The top example (a) represents a high-frequency structure and the bottom example (b) a low-frequency structure. The $3^{\text {rd }}$ level of the parse tree is highlighted with a green dashed box. 


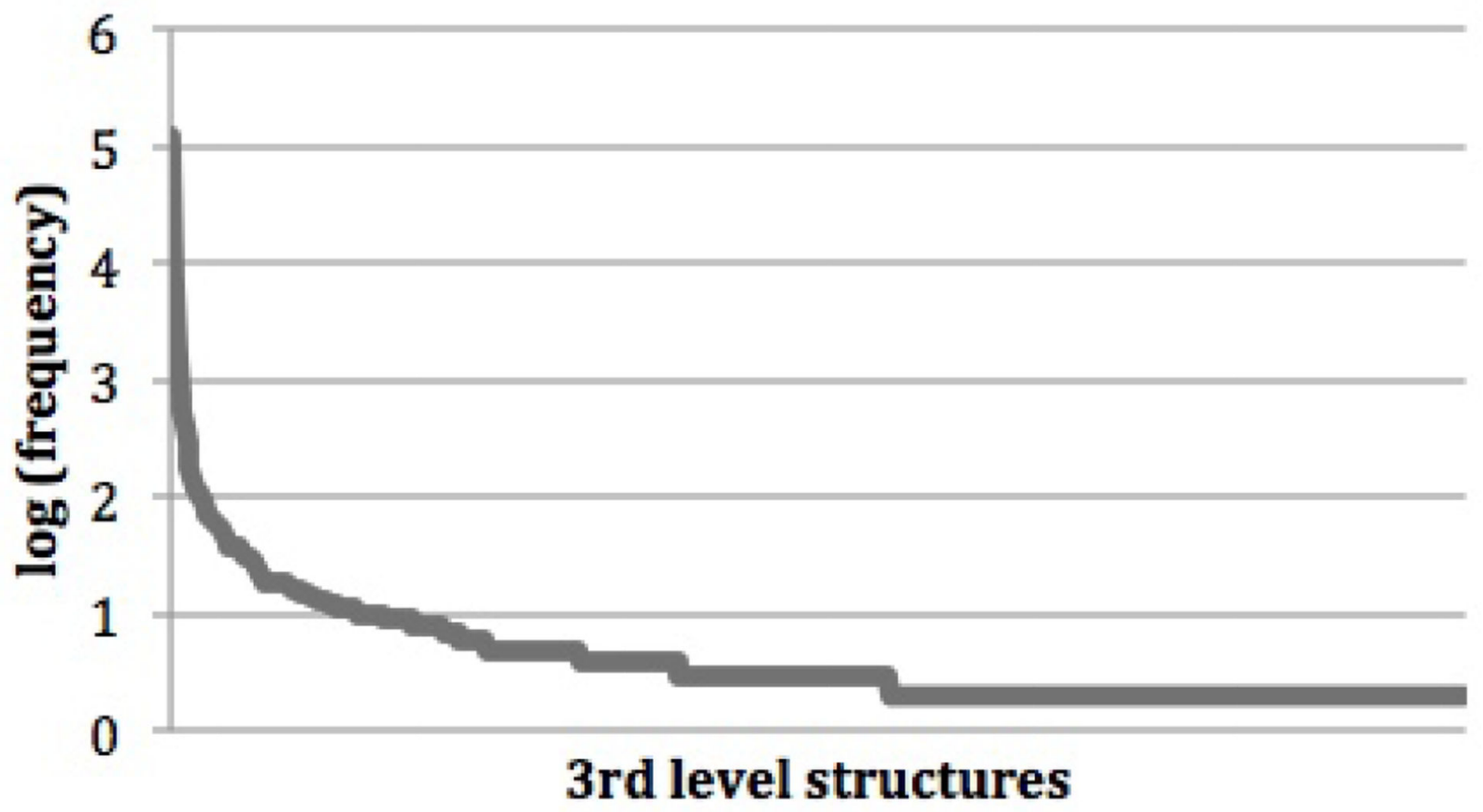

Figure 2.

A plot of the corpus frequency (log-scale) for the $3^{\text {rd }}$ level structures, sorted from most frequent to least. 


\section{However, __ by the rebel ___ to city __ especially ___ soured the welcome.}

a. violence, royalists, residents, army

b. violence, army, residents, royalists

c. residents, army, violence, royalists

d. army, violence, residents, royalists

e. residents, royalists, army, violence

Figure 3.

Example Cloze test task administered to users. 
(a) Actual Difficulty Overall

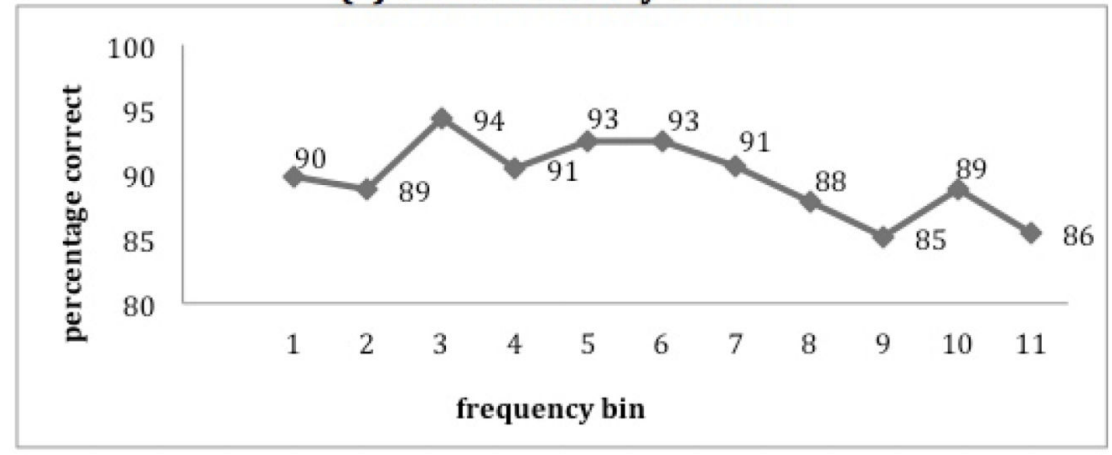

(b) Actual Difficulty by Sentence Length

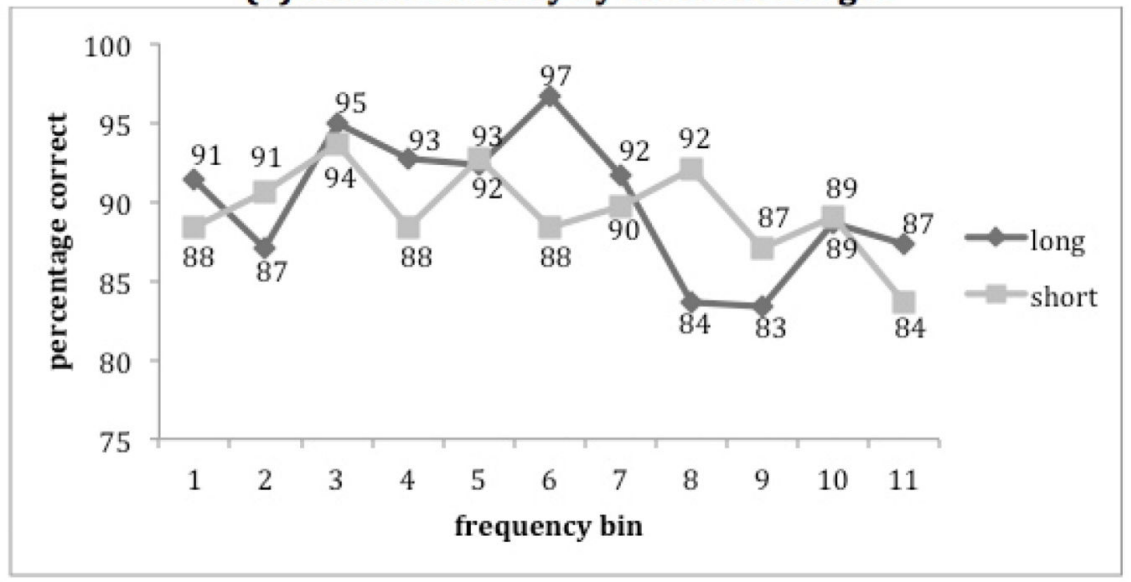

(c) Actual Difficulty by Term Familiarity

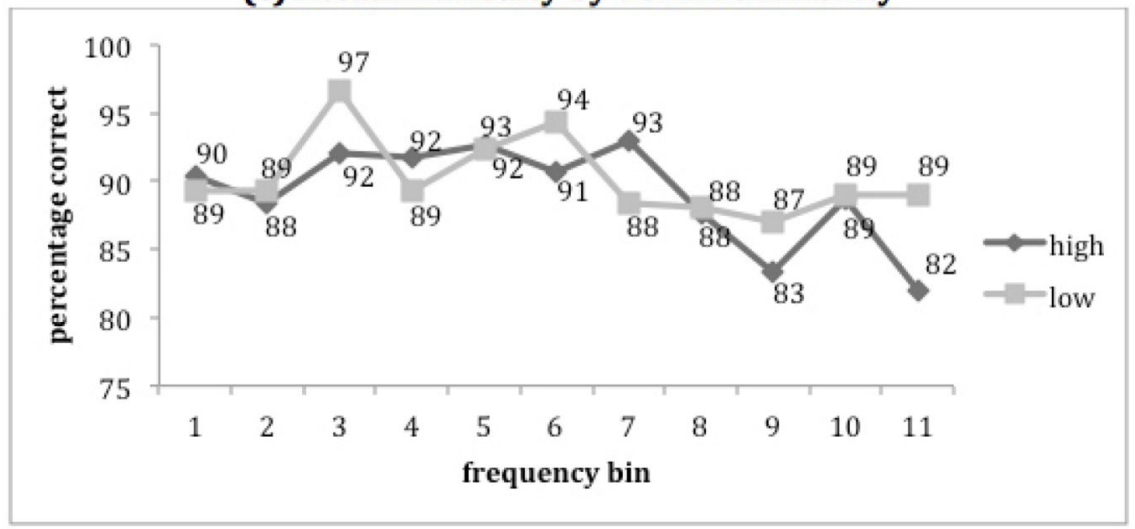

Figure 4.

Percentage correct on the Cloze test (actual difficulty) for the different grammar frequency bins. Higher scores represent better understanding. 
(a) Perceived Difficulty Overall

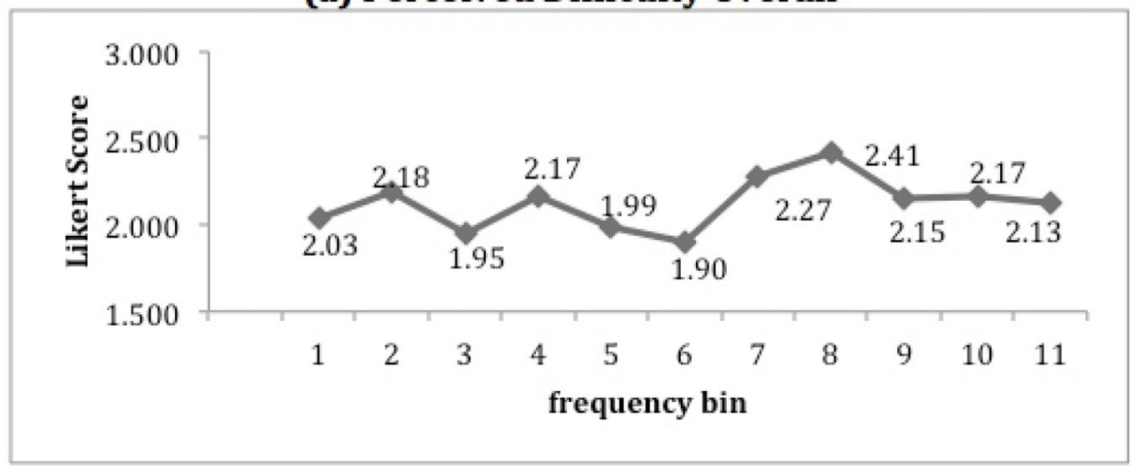

(b) Perceived Difficulty by Sentence Length

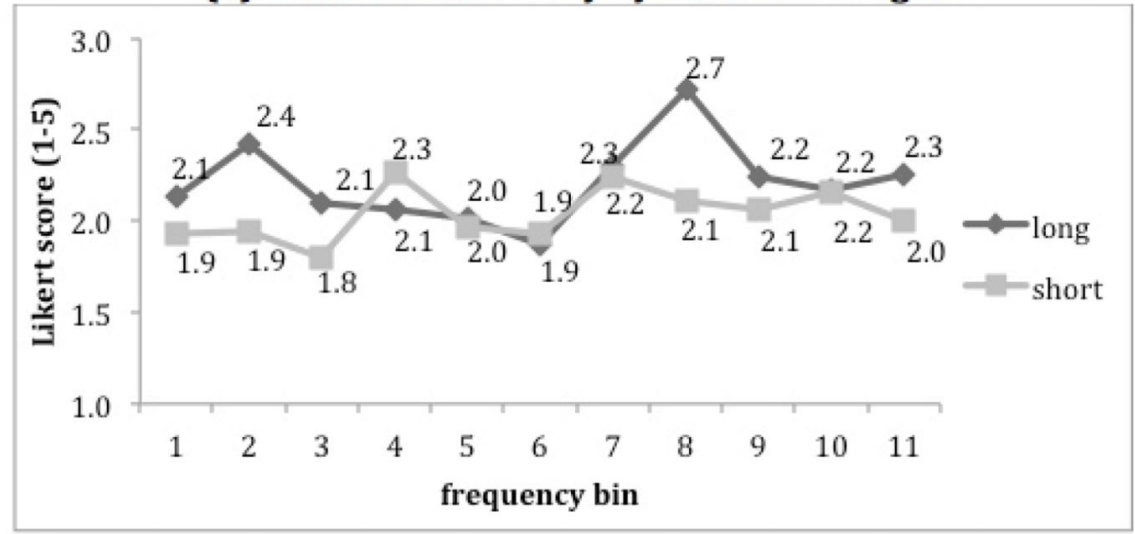

(c) Perceived Difficulty by Term Frequency

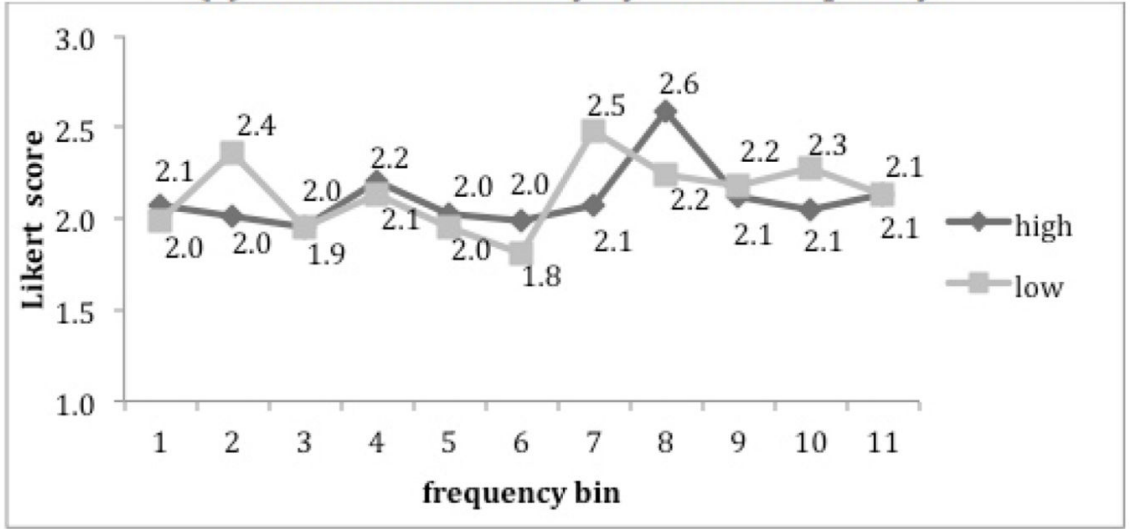

Figure 5.

Perceived difficulty scores for sentences in each of the grammar frequency bins. Scores ranged from 1 to 5 with lower scores representing easier sentences (1: 'Very Easy' - 5: 'Very Difficult'). 


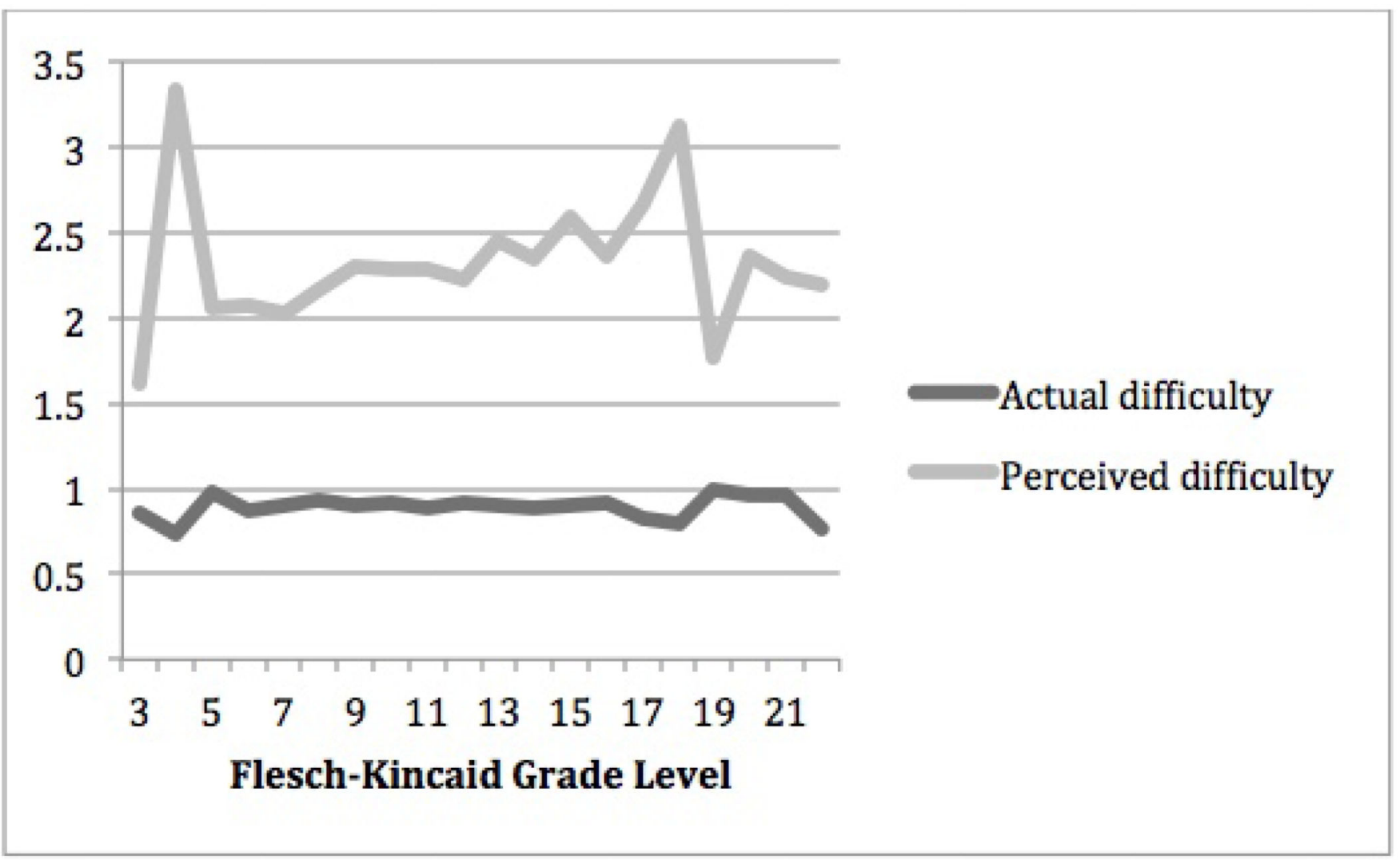

Figure 6.

Actual and perceived difficulty as measured in our user study for the 220 sentences binned by the Flesch-Kincaid grade level. 


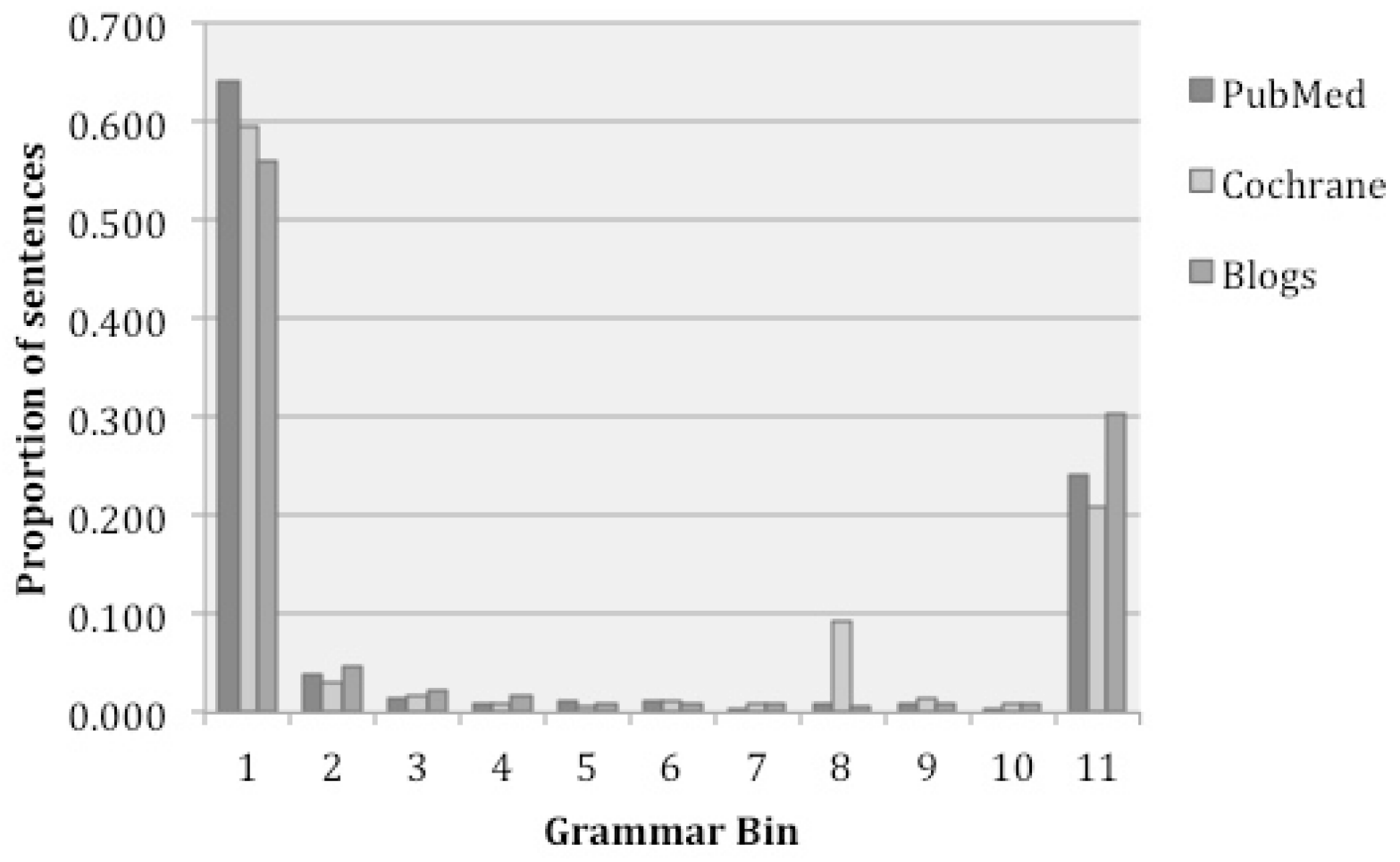

Figure 7.

Proportion of sentences falling into each of the 11 grammar frequency bins for the three corpora. 


\section{Table 1}

Example sentences from the Wikipedia corpus with their 3rd level grammatical structure and the frequency of that structure.

\begin{tabular}{|c|c|c|}
\hline Grammatical Structure & $\begin{array}{c}\text { Corpus } \\
\text { Frequency }\end{array}$ & Example Sentence \\
\hline \multirow[t]{2}{*}{$\begin{array}{l}{[\mathrm{S}[\mathrm{NP}[\mathrm{DT} \mathrm{NN}]]} \\
\quad[\mathrm{VP}[\mathrm{VBZ} \mathrm{NP}]] \\
[. .]]\end{array}$} & \multirow[t]{2}{*}{$\begin{array}{l}24,068 \\
\text { (bin 1) }\end{array}$} & $\begin{array}{l}{\left[[T h e]_{\mathrm{DT}}[\text { painting }]_{\mathrm{NN}}\right]_{\mathrm{NP}}\left[[\mathrm{offers}]_{\mathrm{VPZ}} \text { [a portrayal of the }\right.} \\
\text { single figure of Aaron in his priestly garments } \\
\text { celebrating Yom Kippur in the wilderness } \\
\left.\text { Tabernacle }]_{\mathrm{NP}}\right]_{\mathrm{VP}} \text {. }\end{array}$ \\
\hline & & $\begin{array}{l}{\left[[\text { The }]_{\mathrm{DT}}[\text { foam }]_{\mathrm{NN}}\right]_{\mathrm{NP}}\left[[\text { has }]_{\mathrm{VPZ}} \text { [anti-microbial }\right.} \\
\left.\text { properties }]_{\mathrm{NP}}\right]_{\mathrm{VP}} .\end{array}$ \\
\hline \multirow{2}{*}{$\begin{array}{l}\text { [S [NP [NNP CC NNP] } \\
{[\text { VP [ADVP VBD NP PP] }} \\
[. .]]\end{array}$} & \multirow[t]{2}{*}{$\begin{array}{c}3 \\
\text { (bin 6) }\end{array}$} & $\begin{array}{l}{\left[[\text { Cactus }]_{\mathrm{NNP}}[\text { and }]_{\mathrm{CC}}[\text { Young }]_{\mathrm{NNP}}\right]_{\mathrm{NP}}[\text { briefly }]_{\mathrm{ADVP}}} \\
\left.[\text { held }]_{\mathrm{VBD}}[\text { the CWA tag titles }]_{\mathrm{NP}}[\text { in late } 1988]_{\mathrm{PP}}\right]_{\mathrm{VP}} \text {. }\end{array}$ \\
\hline & & $\begin{array}{l}{\left[[\text { Scotty }]_{\mathrm{NNP}}[\text { and }]_{\mathrm{CC}}[\text { Rikishi }]_{\mathrm{NNP}}\right]_{\mathrm{NP}}[\text { successfully }]_{\mathrm{ADVP}}} \\
{[\text { defended }]_{\mathrm{VBD}} \text { their titles in a four way tag team }} \\
\left.\text { match }]_{\mathrm{NP}}[\text { at WrestleMania XX }]_{\mathrm{PP}}\right]_{\mathrm{VP}} \text {. }\end{array}$ \\
\hline \multirow[t]{2}{*}{$\begin{array}{l}\text { [S [NP [DT JJ JJS NN]] } \\
\quad[\mathrm{VP}[\mathrm{VBZ} \text { NP, S]] } \\
[. .]]\end{array}$} & \multirow[t]{2}{*}{$\stackrel{2}{2}(\operatorname{bin} 11)$} & $\begin{array}{l}{\left[[\text { The }]_{\mathrm{DT}}[\text { closest }]_{\mathrm{JJS}}[\text { English }]_{\mathrm{JJ}}[\text { translation }]_{\mathrm{NN}}\right]_{\mathrm{NP}}[\mathrm{is}]_{\mathrm{VBZ}}} \\
{[\text { academy }]_{\mathrm{NP}} \text {, [referring to institutions of secondary }} \\
\text { learning }]_{\mathrm{VP}} \text {. }\end{array}$ \\
\hline & & $\begin{array}{l}{\left[[\text { The }]_{\mathrm{DT}}[\text { earliest }]_{\mathrm{JJS}}[\mathrm{such}]_{\mathrm{JJ}}[\text { source }]_{\mathrm{NN}}\right]_{\mathrm{NP}}[\mathrm{is}]_{\mathrm{VBZ}} \text { [a life }} \\
\text { of Dunstan by Osbern of Canterbury }]_{\mathrm{NP}} \text {, [probably } \\
\text { written in the } 1080 \mathrm{~s}]_{\mathrm{VP}} \text {. }\end{array}$ \\
\hline
\end{tabular}


Table 2

Example sentences used in this study.

\begin{tabular}{|c|c|c|c|}
\hline $\begin{array}{l}\text { Frequency } \\
\text { Percentile } \\
\text { bin }\end{array}$ & $\begin{array}{l}\text { Sentence } \\
\text { Length }\end{array}$ & $\begin{array}{l}\text { Term } \\
\text { Familiarity }\end{array}$ & Example Sentence \\
\hline \multirow[t]{4}{*}{$\begin{array}{l}\text { Top } 1 \% \\
\left(1^{\text {st }} \text { bin }\right)\end{array}$} & \multirow[t]{2}{*}{ Short } & High & $\begin{array}{l}\text { Soondae, a sausage made of cellophane noodles and pork } \\
\text { blood, is widely eaten. }\end{array}$ \\
\hline & & Low & $\begin{array}{l}\text { Ordination authorizing the deacons of legislation as } \\
\text { women was passed in } 1986 \text { and they were first ordained in } \\
1987 .\end{array}$ \\
\hline & \multirow[t]{2}{*}{ Long } & High & $\begin{array}{l}\text { Mass movement is an important part of the erosional } \\
\text { process, and is often the first stage in the breakdown and } \\
\text { transport of weathered materials in mountainous areas. }\end{array}$ \\
\hline & & Low & $\begin{array}{l}\text { The company installed electrical arc light based } \\
\text { illumination systems designed by Tesla and also had } \\
\text { designs for dynamo electric machine commutators, the } \\
\text { first patents issued to Tesla in the US. }\end{array}$ \\
\hline \multirow[t]{4}{*}{ (6 bin) } & \multirow[t]{2}{*}{ Short } & High & $\begin{array}{l}\text { Unable to defeat him head on, Stark fakes Iron Man's } \\
\text { demise, intending to retire the suit permanently. }\end{array}$ \\
\hline & & Low & $\begin{array}{l}\text { His wife Ashkhen would borrow money from Politburo } \\
\text { wives who had fewer children. }\end{array}$ \\
\hline & \multirow[t]{2}{*}{ Long } & High & $\begin{array}{l}\text { Stone and brick became the city's building materials of } \\
\text { choice after the construction of wood-frame houses was } \\
\text { limited in the aftermath of the Great Fire of } 1835 \text {. }\end{array}$ \\
\hline & & Low & $\begin{array}{l}\text { In the 1990s she again took time from her career to move } \\
\text { into a Romanian hospital to clean cots and wash and care } \\
\text { for the sick and dying orphans. }\end{array}$ \\
\hline \multirow[t]{4}{*}{ (11 $1^{\text {th }}$ bin) } & \multirow[t]{2}{*}{ Short } & High & $\begin{array}{l}\text { However, the CBC continued to program NWI, with much } \\
\text { of its programming simulcast on the domestic Newsworld } \\
\text { service. }\end{array}$ \\
\hline & & Low & $\begin{array}{l}\text { Thus " } 25 \text { th Century" became a track and a } \\
\text { potential band-name became a label. }\end{array}$ \\
\hline & \multirow[t]{2}{*}{ Long } & High & $\begin{array}{l}\text { Also, due to the river inflow, water in the sea has low } \\
\text { salinity and high content of biological matter, such as green } \\
\text { algae that affects the water color. }\end{array}$ \\
\hline & & Low & $\begin{array}{l}\text { An accomplished doubles player, Clijsters is extremely } \\
\text { comfortable at net and mixes up her aggressive-style play } \\
\text { with drop shots and backhand slices to draw her } \\
\text { opponents into the net, where many players are } \\
\text { uncomfortable. }\end{array}$ \\
\hline
\end{tabular}




\section{Table 4}

Summary demographic numbers from study participants.

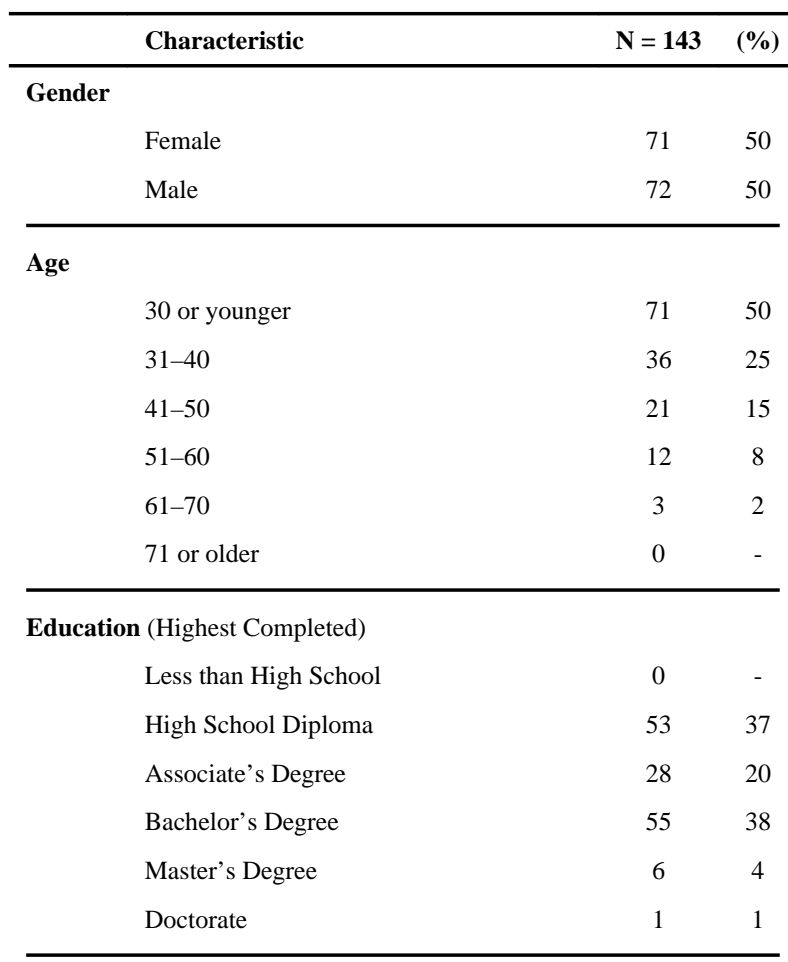

Race (Multiple choices allowed)

American Indian / Native Alaskan $\quad 4 \quad 3$

$\begin{array}{lll}\text { Asian } & 10 & 7\end{array}$

Black or African American $\quad 11 \quad 8$

Native Hawaiian or Other Pacific Islander $\quad 1 \quad 1$

$\begin{array}{lll}\text { White } & 125 & 87\end{array}$

Ethnicity

$\begin{array}{lcc}\text { Hispanic or Latino } & 6 & 4 \\ \text { Not Hispanic or Latino } & 137 & 96\end{array}$




\section{Table 5}

Summary ANOVA results showing the statistical significance of the main effect and the interaction effects for the three evaluation metrics.

\begin{tabular}{|c|c|c|}
\hline Main effect & Interaction effects & p-values \\
\hline \multirow[t]{4}{*}{ Actual Difficulty } & & $\mathrm{p}<0.001$ \\
\hline & sentence length & $\mathrm{p}<0.001$ \\
\hline & term familiarity & $\mathrm{p}=0.044$ \\
\hline & $\begin{array}{l}\text { sentence length and } \\
\text { term familiarity }\end{array}$ & $\mathrm{p}<0.001$ \\
\hline \multirow[t]{4}{*}{ Perceived Difficulty } & & $\mathrm{p}<0.001$ \\
\hline & sentence length & $\mathrm{p}<0.001$ \\
\hline & term familiarity & $\mathrm{p}<0.001$ \\
\hline & $\begin{array}{l}\text { sentence length and } \\
\text { term familiarity }\end{array}$ & $\mathrm{p}<0.001$ \\
\hline \multirow[t]{4}{*}{ Time } & & $\mathrm{p}<0.001$ \\
\hline & sentence length & $\mathrm{p}<0.001$ \\
\hline & term familiarity & not significant \\
\hline & $\begin{array}{l}\text { sentence length and } \\
\text { term familiarity }\end{array}$ & $\mathrm{p}=0.030$ \\
\hline
\end{tabular}




\section{Table 6}

Size, in number of text and number of sentences, of the three corpora analyzed using grammar frequency bins.

\begin{tabular}{c|c|c} 
Source & Number of texts & Number of sentences \\
\hline PubMed & 36,067 & 360,123 \\
\hline Cochrane & 2,641 & 44,488 \\
\hline Blogs & 139 & 5,657 \\
\hline
\end{tabular}

